\title{
LIMK1/TPPP1/HDAC6 Is a Dual Actin and Microtubule Regulatory Complex That Promotes Drug Resistance
}

\author{
Alice V. Schofield ${ }^{1,2}$, Cristina Gamell1, Ora Bernard ${ }^{1,2 *}$ \\ ${ }^{1}$ St Vincent's Institute of Medical Research, Melbourne, Australia \\ ${ }^{2}$ Department of Medicine, The University of Melbourne, St Vincent's Hospital, Melbourne, Australia \\ Email: ${ }^{*}$ obernard@svi.edu.au
}

Received 16 January 2014; revised 17 February 2014; accepted 5 March 2014

Copyright (C) 2014 by authors and Scientific Research Publishing Inc.

This work is licensed under the Creative Commons Attribution International License (CC BY). http://creativecommons.org/licenses/by/4.0/

(c) (i) Open Access

\begin{abstract}
In this study, we identified a novel protein complex consisting of LIM-Kinase 1 (LIMK1), Histone deacetylase 6 (HDAC6) and Tubulin Polymerization Promoting Protein 1 (TPPP1). Under basal conditions, assembly of the LIMK1/TPPP1/HDAC6 complex results in both inhibition of HDAC6 activity and LIMK1 activation. This leads to increased microtubule (MT) acetylation, a MT stabilizing modification, and actin filament (F-actin) destabilization. In response to activation of the Rhokinase (ROCK) signaling pathway, downstream phosphorylation of LIMK1 and TPPP1 leads to the dissociation of the LIMK1/TPPP1/HDAC6 complex. In turn, HDAC6 and LIMK1 activities are increased, which results in MT destabilization and F-actin stabilization. Finally, we reveal that increasing tubulin acetylation reduces the efficacy of chemotherapeutic drugs, suggesting that strategies to reduce acetyl-tubulin levels may be a viable option in treating drug-resistant tumors.
\end{abstract}

\section{Keywords}

Actin; Deacetylase; Drug Sensitivity; Kinase; Microtubules

\section{Introduction}

The LIM-kinase (LIMK) family of proteins kinases including LIMK1 and LIMK2, promote actin polymerization by phosphorylation and inhibition of the actin depolymerizing and severing proteins cofilin/ADF [1] [2]. LIMKs are activated through phosphorylation by Rho-associated coiled-coil kinase (ROCK), p21-activated ki-

\footnotetext{
*Corresponding author.
}

How to cite this paper: Schofield, A.V., Gamell, C. and Bernard, O. (2014) LIMK1/TPPP1/HDAC6 Is a Dual Actin and Microtubule Regulatory Complex That Promotes Drug Resistance. Advances in Bioscience and Biotechnology, 5, $353-362$. 
nase 1 (PAK1) and PAK4, downstream effectors of the small GTPases Rho, Rac and Cdc42, respectively [3]-[5]. Interestingly, although it has been reported that LIMK1 and LIMK2 interact with Tubulin Polymerization Promoting Protein 1 (TPPP1) in vitro and in vivo [6]-[8], TPPP1 is not a LIMK substrate in cells [9].

TPPP1 is a small protein that regulates microtubule (MT) dynamics, through two different mechanisms to promote MT polymerization; first by increasing MT polymerization kinetics and second by inhibiting the activity of Histone deacetylase 6 (HDAC6), resulting in increased MT acetylation and stabilization [10] [11]. We have recently reported that similar to the LIMKs, TPPP1 is also a substrate of ROCK and that ROCK-mediated TPPP1 phosphorylation inhibits its ability to bind and inhibit HDAC6 activity [9].

In the present study, we identified the formation of a LIMK1/TPPP1/HDAC6 complex in cells. The complex formation leads to inhibition of HDAC6 and LIMK1 activities thereby increasing microtubule (MT) acetylation, and reducing actin filament polymerization by inhibiting LIMK1-mediated cofilin phosphorylation. We reveal that through increasing tubulin acetylation levels the complex reduces the efficacy of MT-targeted chemotherapeutic drugs, thereby suggesting that strategies to reduce acetyl-tubulin levels may be a viable option in treating drug-resistant tumors.

\section{Materials and Methods}

\subsection{Plasmid Constructs}

pBABE-Flag-TPPP1 [6], pBABE-Flag-LIMK1, pBABE-FLag-LIMK1-DN [12], myc-cofilin, myc-cofilin S3A and myc-cofilin S3D [13] plasmids were generated as previously described. pcDNA3-Flag-HDAC6 was obtained from Addgene and was originally cloned as described [14].

\subsection{Mammalian Cell Culture}

U2OS (human osteosarcoma) cells were cultured and maintained in Dulbecco's Modified Eagle Media (DMEM) supplemented with $10 \%$ Fetal Bovine Serum (FBS) at $37^{\circ} \mathrm{C}$ in a humidified, $5 \% \mathrm{CO}_{2}$ atmosphere. Flag-LIMK1, Flag-LIMK1-DN, Flag-HADC6, myc-cofilin, myc-cofilin S3A, myc-cofilin S3D constructs were transiently transfected into U2OS cells using the Lipofectamine ${ }^{\mathrm{TM}} 2000$ (Life Technologies) transfection reagent according to manufacturer's recommendations. Cells expressing Flag-TPPP1 were generated as previously described [9].

\subsection{Reagents and Treatments}

The following chemicals were obtained and used in this study: Y-27632 (Calbiochem), Paclitaxel (Cytoskeleton), Vincristine (Gift of Dr Maria Kavallaris, Lowy Cancer Research Centre, Sydney) and Trichostatin A (Gift of Dr Boris Sarcevic, St. Vincent's Institute, Melbourne). Treatment of cells with Y-27632 (10 $\mu \mathrm{M})$, Paclitaxel (500 nM), Vincristine (100 nM) and Trichostatin A (100 nM) were performed for 16 hours.

\subsection{RNA Interference Assays}

Cells were transfected with hTPPP1 ON-TARGETplus SMARTpool, hLIMK1 siRNA 5'-TGGCAAGCGTGGACTTTCA-3' oligonucleotides or non-targeting siRNA (Dharmacon) using the Lipofectamine ${ }^{\mathrm{TM}} 2000$ (Life Technologies) transfection reagent according to the manufacturer's recommendations.

\subsection{Immunoprecipitation Studies}

U2OS cell extracts $(500 \mu \mathrm{g})$ were pre-cleared with $\sim 2 \mu \mathrm{g}$ of the appropriate isotype control antibodies (anti-mouse, rabbit or rat IgG) and $30 \mu \mathrm{L}$ of Protein A (Rabbit IgG) or Protein G (Rat and Mouse IgG) sepharose beads for 2 hours at $4^{\circ} \mathrm{C}$. Cleared lysates were incubated with $\sim 2 \mu \mathrm{g}$ of isotype control, anti-HDAC6 (Sigma-Aldrich), anti-TPPP1 [6] or anti-LIMK1 [12] antibodies and Protein A/G sepharose overnight at $4^{\circ} \mathrm{C}$ with rotation. Immunoprecipitated (IP) proteins were resolved by SDS-PAGE and subjected to immunoblot analysis.

\subsection{Immunoblotting}

Immunoblotting was performed as previously described [9]. Antibodies against phospho-cofilin (Ser3; \#3311), phospho-MLC (Ser18, Thr19; \#3674) and GAPDH-HRP (\#3683) were obtained from Cell Signaling. An- 
ti- $\alpha$-tubulin (T5168), acetyl- $\alpha$-tubulin (Lys40; T6793) and HDAC6 (SAB1406911) antibodies were purchased from Sigma-Aldrich. Additional antibodies used were TPPP1 [6], LIMK1 [12], LIMK2 [15], Flag 9H1 clone [16] and c-myc tag clone 4A6 (Upstate). Immunoblot quantification was performed using the ImageQuant software. Data are expressed as mean \pm S.E.M and sample groups were analyzed by unpaired two-tailed t-tests.

\subsection{Immunofluorescence Microscopy}

Immunofluorescence was performed as previously described [9]. Cells were incubated with anti-acetyl- $\alpha$-tubulin (1:1000; Sigma-Aldrich) followed by anti-mouse IgG Alexa Fluor 488 (1:400; Life Technologies), anti- $\alpha$ tubulin-FITC (1:400; Sigma-Aldrich, F2168) or Phalloidin Alexa Fluor 488 (1:400; Life Technologies, A12379) and Hoechst (1:10,000; Life Technologies). Images were acquired using a Zeiss AxioObserver LSM 780 inverted microscope with a 63x/1.46 oil immersion DICIII lens. Files were extracted using the Image J (v1.47) software. Image overlay was performed with the Adobe Photoshop (v11.0.2) software.

\subsection{MTT Assays}

Cells were plated in triplicate on 96-well plates and 24 hours later they were incubated with the MTT reagent (CellTiter $96^{\circledR}$ AQueous Non-Radioactive Cell Proliferation Assay kit (Promega)) for 2 hours at $37^{\circ} \mathrm{C}$ in $5 \%$ $\mathrm{CO}_{2}$. Converted MTT dye was analyzed at OD490 nm ( $\lambda$ 490) using a spectrophotometer plate reader (Titertek Multiskan Plus, Lab Systems). Data are expressed as mean \pm S.E.M and sample groups were analyzed by unpaired two-tailed t-tests.

\section{Results and Discussion}

\subsection{LIMK1/TPPP1/HDAC6 Form a Trimeric Complex}

Previous studies reported that the LIM-kinase (LIMK) family members LIMK1 and LIMK2 interact with the microtubule regulatory protein Tubulin Polymerization Promoting Protein 1 (TPPP1) [6] [8] [17]. Contrary to the initial data indicating that TPPP1 is an in vitro LIMK substrate [6] [17], we established that the LIMKs do not phosphorylate TPPP1 and that it is a substrate of ROCK in cells [9]. TPPP1 interacts with and inhibits Histone deacetylae 6 (HDAC6) activity [9] [11]. Given the proximal interactions between LIMK1/TPPP1 and TPPP1/HDAC6, we hypothesized that LIMK1, TPPP1 and HDAC6 form a complex. Immunoprecipitation of LIMK1, TPPP1 or HDAC6 from U2OS osteosarcoma cells demonstrated that they reciprocally co-precipitated each other, indicating that they form a trimeric complex (Figures 1(a)-(c)).

LIMK1 and TPPP1 are both substrates of ROCK [3] [9]. To investigate the impact of LIMK1 and TPPP1 phosphorylation on the stability of the LIMK1/TPPP1/HDAC6 complex, we performed immunoprecipitation studies with U2OS cell lysates that were treated with the ROCK inhibitor Y-27632 $(10 \mu \mathrm{M})$ or vehicle. Our results demonstrate that decreased ROCK signaling (Figure 1(d)) resulting in reduced LIMK1 and TPPP1 phosphorylation increased the interaction between LIMK1/TPPP1/HDAC6 (Figures 1(e)-(g)). Further analysis of the complex dynamics revealed that stimulation of ROCK activity by treatment of cells with FBS (10\%) (Figure 1(h)) reduced their interaction (Figures $1(\mathrm{~g})-(\mathrm{k}))$. Therefore, these results demonstrate that LIMK1, TPPP1 and HDAC6 form a trimeric complex under basal conditions, and that activation of ROCK signaling, resulting in LIMK1 and TPPP1 phosphorylation, reduces LIMK1/TPPP1/HDAC6 interaction.

\subsection{Cellular Expression of LIMK1 and TPPP1 Regulates Tubulin Acetylation and LIMK1 Activity, Respectively}

TPPP1/HDAC6 interaction results in the inhibition of HDAC6 activity [9]. Since HDAC6 catalyzes the deacetylation of Lys40 on $\beta$-tubulin, the TPPP1/HDAC6 interaction promotes microtubule (MT) stability via increasing its acetylation. Since LIMK1, TPPP1 and HDAC6 are associated in cells, we examined the possibility that altered LIMK1 expression modulates tubulin acetylation in cells. Analysis of cells overexpressing Flag-LIMK1 revealed a significant increase in acetyl-tubulin levels (Figures 2(a) and (b)). Conversely, the analysis of cells treated with LIMK1 siRNA revealed that reduced LIMK1 expression resulted in decreased acetyl-tubulin levels (Figures 2(c) and (d)), similar to our previously published observations with LIMK2 [8]. Therefore, our results demonstrate that LIMK1 is an integral component of the complex, which along with TPPP1 is required to inhibit 


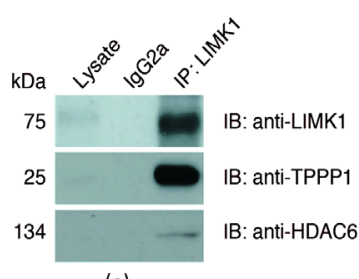

(a)

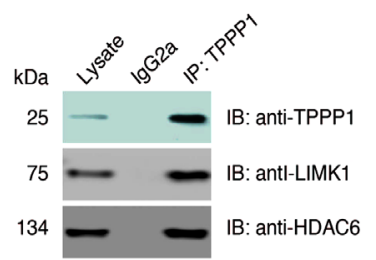

(b)

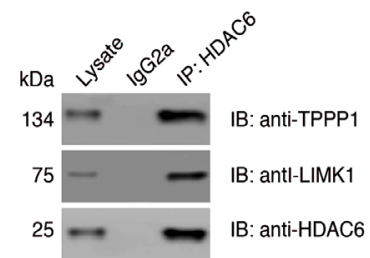

(c)

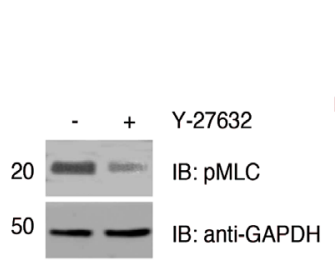

(d)

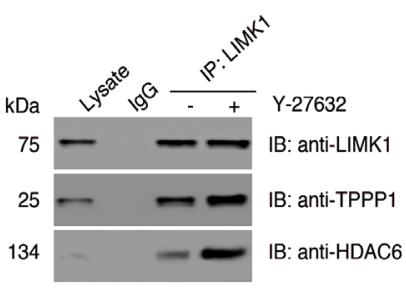

(e)

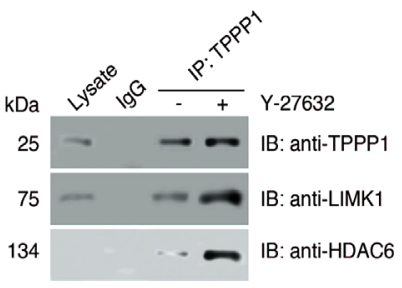

(f)

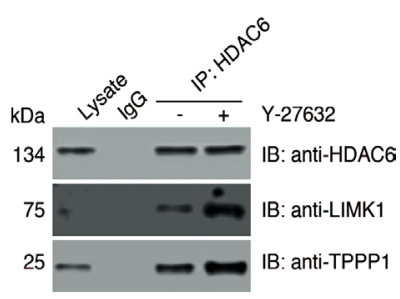

(g)

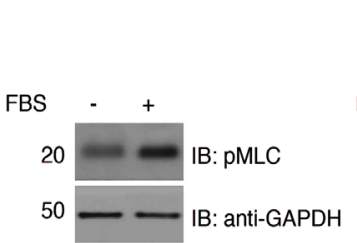

(h)

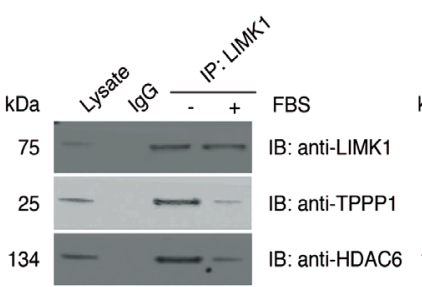

(i)

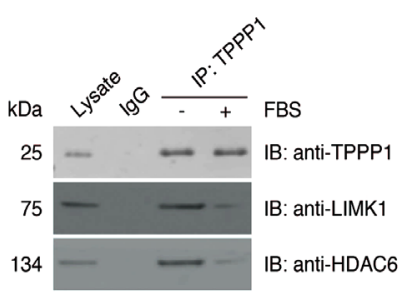

(j)

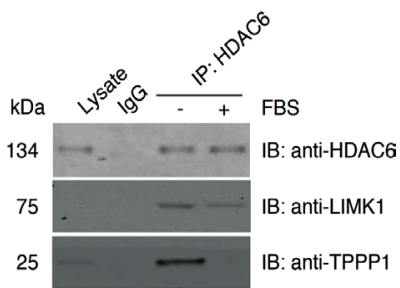

(k)

Figure 1. LIMK1, TPPP1 and HDAC6 form a trimeric complex that is inhibited by ROCK signaling. (a)-(c) U2OS cell lysates immunoprecipitated with LIMK1, TPPP1 or HDAC6 and IgG control antibodies and immunoblotted for their interaction with each other. (d)-(g) Inhibition of ROCK activity (d) increases the trimeric LIMK1/TPPP1/HDAC6 complex interaction. U2OS cells treated with Y-27632 $(10 \mu \mathrm{M})$ or vehicle for 16 hrs were immunoprecipitated and analyzed as described in (a)-(c). (H-K) Activation of ROCK signaling (H) inhibits the LIMK1/TPPP1/HDAC6 complex formation. Serum starved U2OS cells stimulated with $10 \%(+)$ or $0.1 \%(-)$ FBS were immunoprecipitated and analyzed as described in (a)-(c).

\section{HDAC6 activity.}

Our findings suggest that the dynamics of the complex is determined by the level of the components, particularly the un-phosphorylated forms of LIMK1 and TPPP1. Therefore, we hypothesized that the trimer may also modulate LIMK1 activity by protecting it from phosphorylation and therefore its activation. To test this possibility, we analyzed the level of cofilin phosphorylation, the major substrate of LIMK1, in cells expressing altered TPPP1 levels. Analysis of cells overexpressing Flag-TPPP1 revealed that increased TPPP1 expression resulted in a reduction in cofilin phosphorylation (Figure 2(e)) as well as a decrease in cellular filamentous actin (F-actin) levels (Figure 2(f)). Furthermore, analysis of cells transiently transfected with TPPP1 siRNA revealed that knockdown of TPPP1 resulted in an increase in cofilin phosphorylation (Figure 2(g)) and F-actin levels (Figure 2(h)).

Thus far, our results indicate that the LIMK1/TPPP1/HDAC6 trimer autonomously regulates actin and microtubule morphology. However, the inter-cytoskeletal arrangement of actin and microtubules in cells suggests that the downstream effects of the complex may be the result of cross talk between the two networks. To rule out this possibility, we analyzed tubulin acetylation levels in cells overexpressing wild-type myc-cofilin, myc-cofilin S3A (phospho-inhibitory), myc-cofilin S3D (phospho-mimetic) or vector. We found that changes in cofilin activity did not alter tubulin acetylation in cells (Figures 3(a) and (b)). Similarly, we investigated the possibility that differences in tubulin stability may affect cofilin phosphorylation and therefore have an impact on the actin cytoskeleton. For this, we analyzed cofilin phosphorylation levels in cells overexpressing HDAC6 or treated with the HDAC inhibitor Trichostatin A (TSA). We show that neither increased HDAC6 expression (Figures 3(c) and (d)) nor reduced HDAC activity (Figures 3(e) and (f)) altered cofilin phosphorylation or F-actin levels in cells. Overall, these results demonstrate that the LIMK1/TPPP1/HDAC6 trimer has a dual role of inhibiting HDAC6 activity and preventing LIMK1 activation to increase MT acetylation and stability as well as increasing cofilin activity and actin depolymerization. 


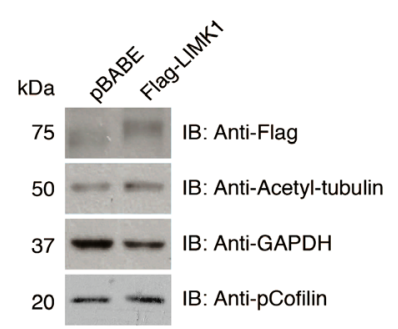

(a)

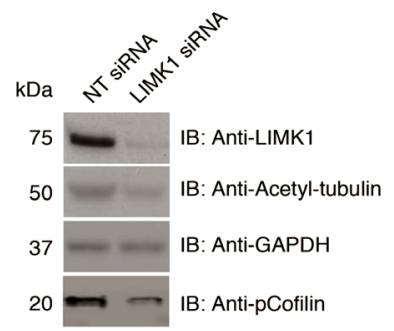

(c)

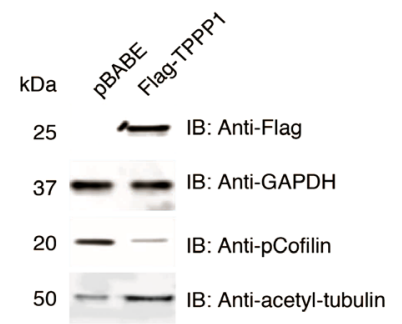

(e)

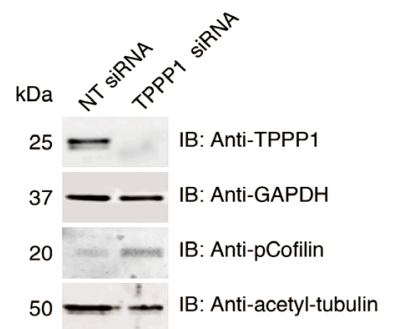

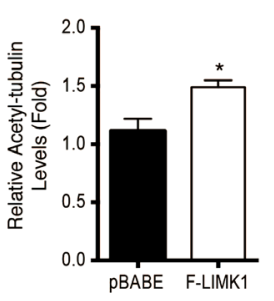
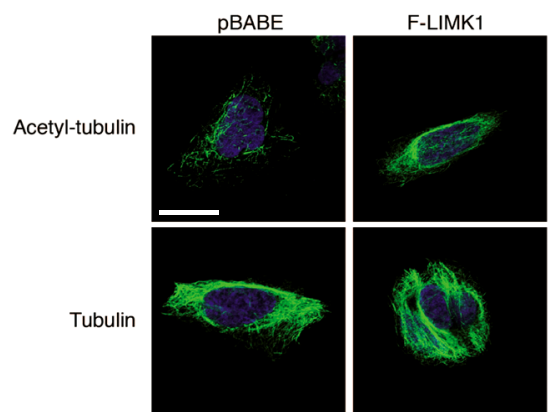

(b)

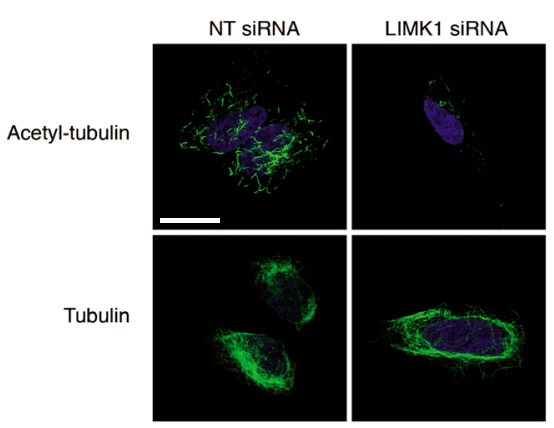

(d)
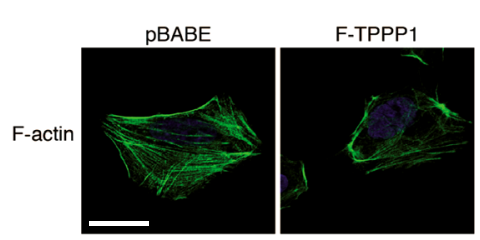

(f)

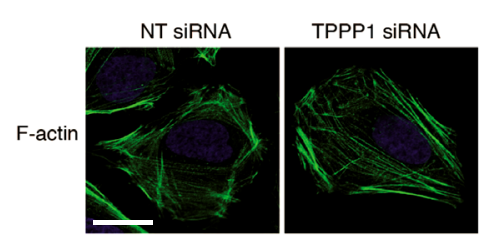

(h)

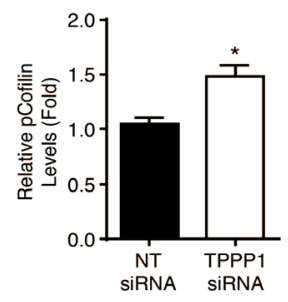

(g)

Figure 2. The LIMK1/TPPP1/HDAC6 trimer regulates tubulin acetylation and LIMK1 activity. (a) LIMK1 overexpression increases acetyl-tubulin levels in cells. Cells transiently transfected with Flag-LIMK1 or vector were analyzed by immunoblotting with anti-Flag, acetyl-tubulin, p-cofilin and GAPDH (loading control) antibodies. Densitometry analysis of immunoblots demonstrated that cells expressing Flag-LIMK1 displayed a significant increase in acetyl-tubulin levels compared to control ( ${ }^{*} \mathrm{p}<0.05$; $n=3$ ). (b) Immunofluorescence microscopy analysis of acetyl-tubulin and tubulin levels in cells described in (a). (c) Knockdown of LIMK1 reduces acetyl-tubulin levels in cells. U2OS cells transiently tranfected with LIMK1 or non-targeting (NT) siRNA control were analyzed as described in (a). (d) Cells described in (c) were analyzed as in (b). (e) TPPP1 overexpression decreases cofilin phosphorylation in cells. U2OS cells transiently transfected with Flag-TPPP1 or vector control were analyzed by immunoblotting with anti-Flag, acetyl-tubulin, p-cofilin or GAPDH (loading control) antibodies and analyzed as described in (a). ( ${ }^{*} \mathrm{p}<0.05 ; \mathrm{n}=3$ ). (f) Immunofluorescence microscopy analysis of F-actin levels by staining cells with Alexa Fluor 488-Phalloidin of cells described in (a). (g) TPPP1 knockdown increases cofilin phosphorylation in cells. U2OS cells were transiently transfected with TPPP1 or NT siRNA and analyzed as described in (e). (h) Cells described in (g) were analyzed as described in (f). Data are expressed as mean \pm SEM. Scale bar $=50 \mu \mathrm{m}$. 

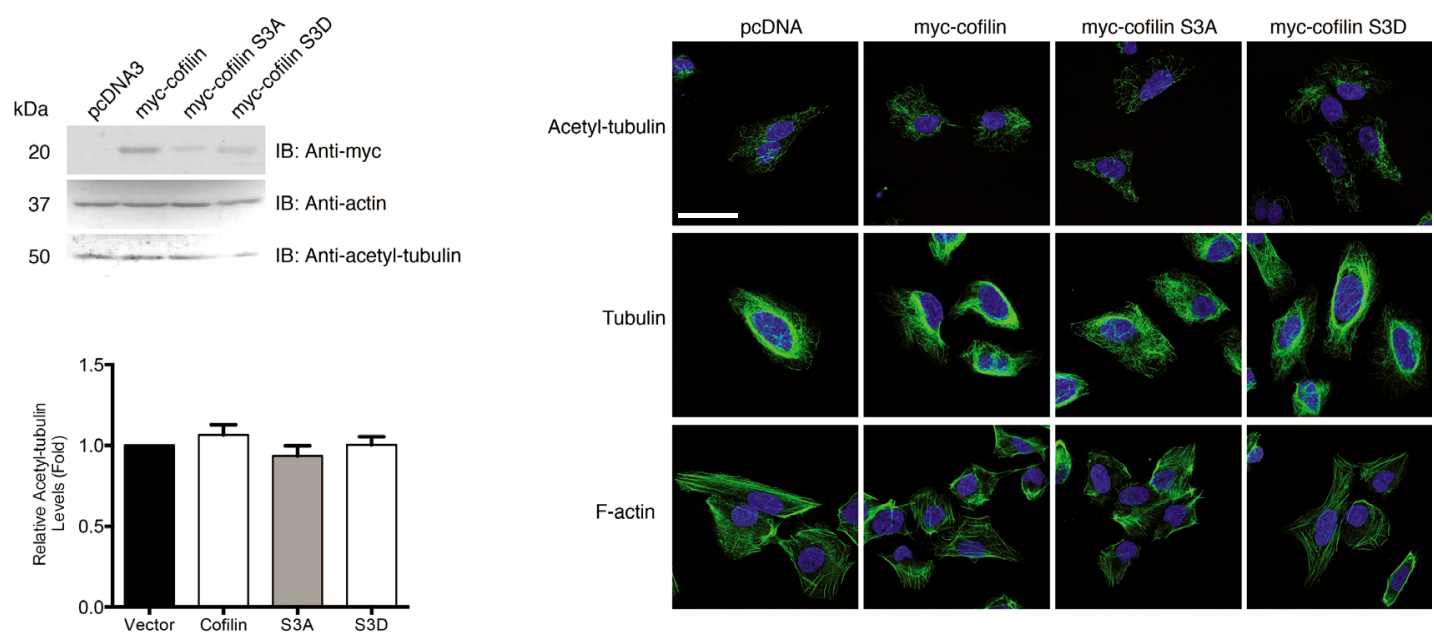

(a)

(b)
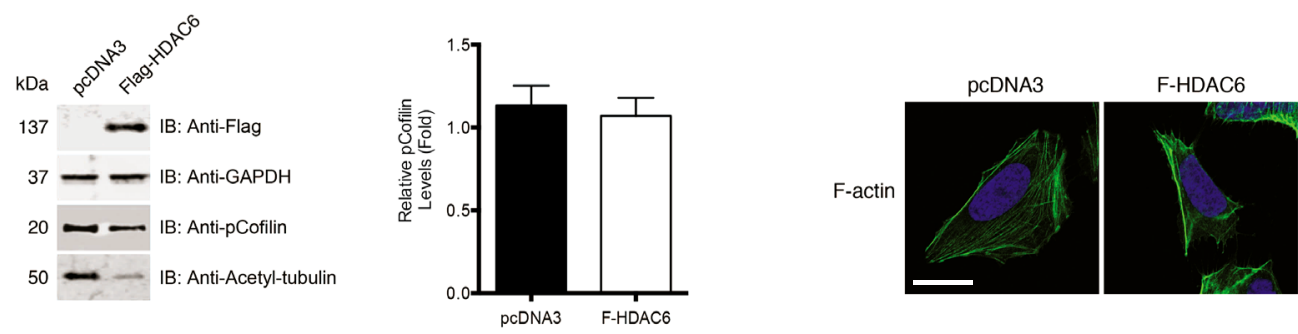

(c)

(d)
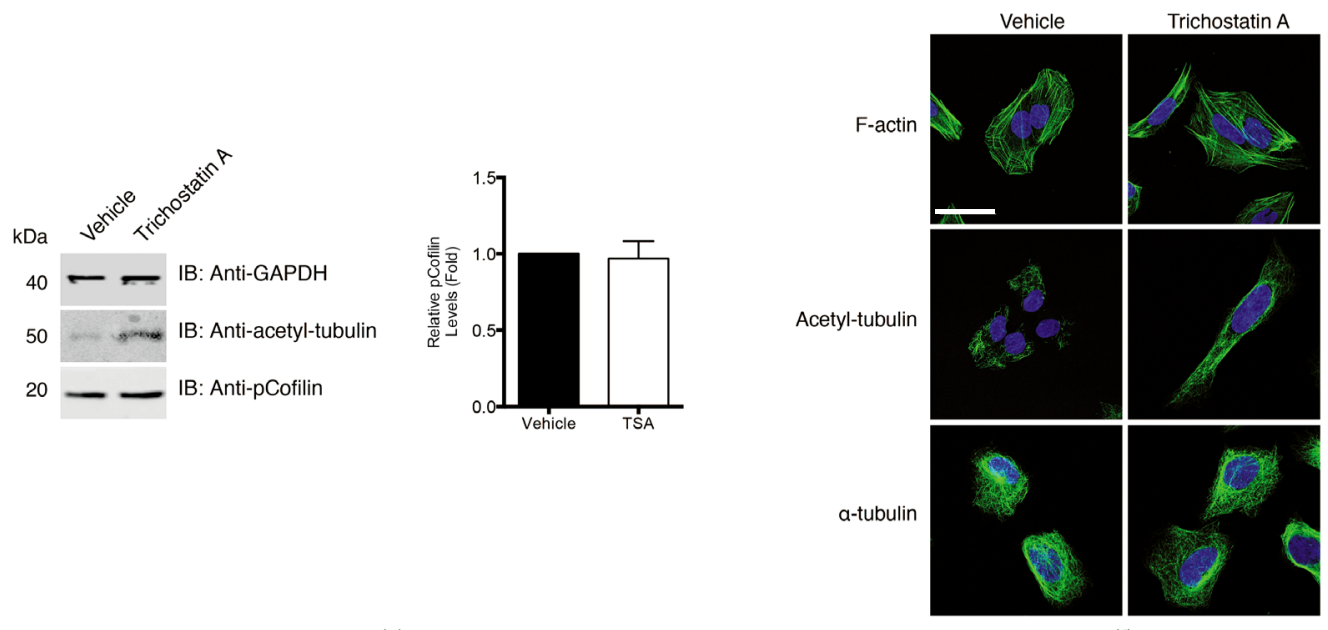

Figure 3. The LIMK1/TPPP1/HDAC6 complex independently regulates Tubulin acetylation and F-actin levels in cells. (a) Overexpression of myc-cofilin does not alter tubulin acetylation in cells. U2OS cells were transiently transfected with myc-cofilin, myc-cofilin S3A (phospho-inhibitory), myc-cofilin S3D (phospho-mimetic) or vector and analyzed by immunoblotting with anti-myc, acetyl-tubulin or GAPDH (loading control) antibodies $(\mathrm{n}=3$ ). (b) Immunofluorescence microscopy analysis of cells described in (a). (c) HDAC6 overexpression does not alter cofilin phosphorylation or F-actin levels. U2OS cells transiently transfected with Flag-HDAC6 were analyzed by immunoblotting with anti-Flag, pCofilin, acetyl-tubulin and GAPDH (loading control). (d) Cells described in (c) were analyzed by immunofluorescence microscopy for F-actin. (e) Inhibition of HDAC activity does not alter cofilin phosphorylation or F-actin levels in cells. U2OS cells were treated with Trichostatin A (TSA; $100 \mathrm{nM}$ ) or vehicle (DMSO) and analyzed by immunoblotting with anti-acetyl-tubulin, pCofilin or GAPDH (loading control) antibodies. (f) Immunofluorescence microscopy analysis of cells described in (e). Data are expressed as mean \pm SEM. Scale bar $=50 \mu \mathrm{m}$. 


\subsection{The LIMK1/TPPP1/HDAC6 Complex Modulates Chemotherapeutic Drug Sensitivity}

The microtubule network undergoes dynamic polymerization and depolymerization according to the cells functional requirements. These dynamics are interrupted by chemotherapeutic agents such as vincristine and paclitaxel to enforce cell cycle checkpoints and ultimately initiate cell death programs. Our results demonstrate that the LIMK1/TPPP1/HDAC6 complex promotes MT stabilization. Therefore, we hypothesized that via increasing MT rigidity the complex could reduce the efficacy of the chemotherapeutic agents vincristine and paclitaxel, MT destabilizing and stabilizing compounds, respectively. Analysis of cell viability demonstrated that overexpression of LIMK1 or TPPP1 reduced vincristine- and paclitaxel-induced cell death compared to control (Figures 4(a) and (c)), whereas siRNA-mediated knockdown of LIMK1 or TPPP1 increased drug-induced cell death compared to control (Figures 4(b) and (d)). These results suggest that reduced acetyl-tubulin levels correlate with increased sensitivity to chemotherapeutic agents. To further test this possibility we overexpressed HDAC6 and analyzed vincristine- and paclitaxel-induced cell death. We show that reduced acetyl-tubulin levels sensitize cells to drug-induced cell death (Figure 4(e)).

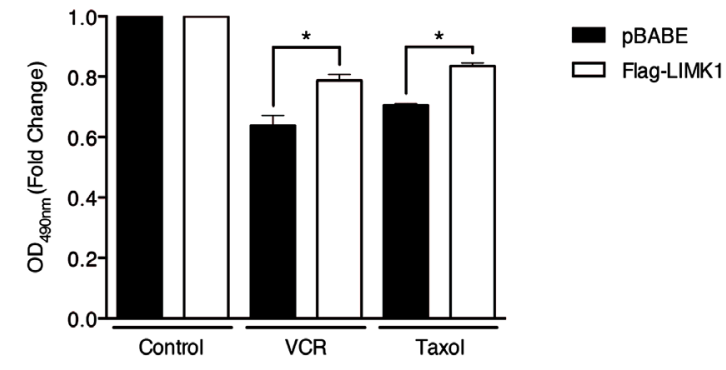

(a)

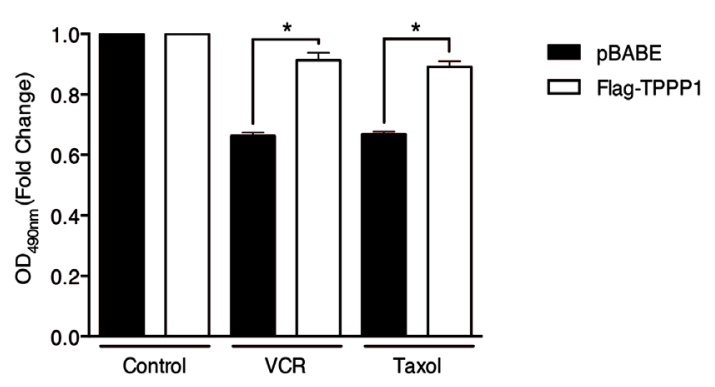

(c)

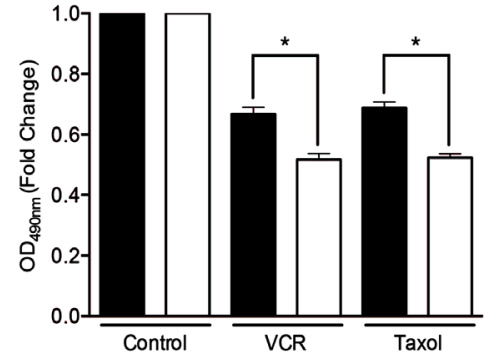

(b)

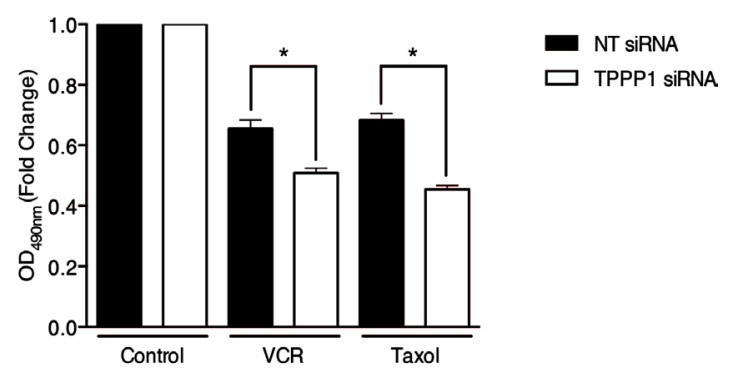

(d)

$$
\begin{aligned}
& \text { NT siRNA } \\
& \text { LIMK1 siRNA }
\end{aligned}
$$

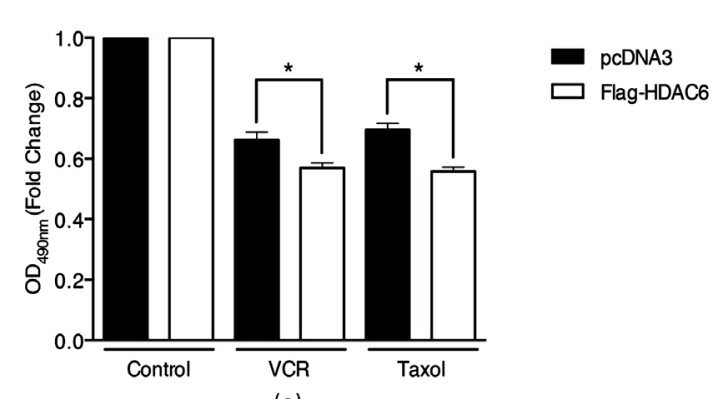

(e)

Figure 4. LIMK1/TPPP1/HDAC6 inhibits chemotherapy-induced cell death by modulation of tubulin acetylation. LIMK1 overexpression and knockdown decrease and increase paclitaxel and vincristine-induced cell death, respectively. (a) U2OS cells expressing Flag-LIMK1 or vector were treated with vincristine (VCR, $100 \mathrm{nM}$ ) or paclitaxel (500 nM) and cell viability was analyzed using an MTT assay. (b) Cells transiently transfected with LIMK1 or NT siRNA were treated and analyzed as described in (a). TPPP1 overexpression and knockdown decrease and increase vincristine- and paclitaxel-induced cell death, respectively. (c) U2OS cells expressing Flag-TPPP1 or vector were treated and analyzed as described in (a). (d) Cells transiently transfected with TPPP1 or NT siRNA were treated and analyzed as described in (a). (e) HDAC6 overexpression increases vincristine- and paclitaxel-induced cell death. U2OS cells expressing Flag-HDAC6 were treated and analyzed as described in (a). Data are expressed as mean \pm SEM. 


\section{Conclusion}

We identify a novel protein complex consisting of LIMK1, TPPP1 and HDAC6 that simultaneously regulate cellular MT and actin filament morphology. The complex is formed in the absence of ROCK signaling pathway activation and results in reduced LIMK1 activity, leading to decreased F-actin levels in cells as well as HDAC6 inhibition and consequential increased MT acetylation and stabilization. Upon activation of the ROCK signaling pathway, phosphorylation of LIMK1 and TPPP1 results in disassembly of the complex. Under these conditions LIMK1-mediated cofilin phosphorylation and inactivation is increased, therefore driving F-actin accumulation as well as increased HDAC6 activity resulting in reduced MT acetylation and stability (Figure 5). Furthermore, we demonstrate that the complex, via modulation of MT acetylation, regulates cellular sensitivity to the chemotherapeutic agents vincristine and paclitaxel. Our findings suggest that strategies to reduce MT acetylation, such as inhibition of acetyl-transferase enzymes, could be used to sensitize tumor cells to chemotherapeutic drugs.

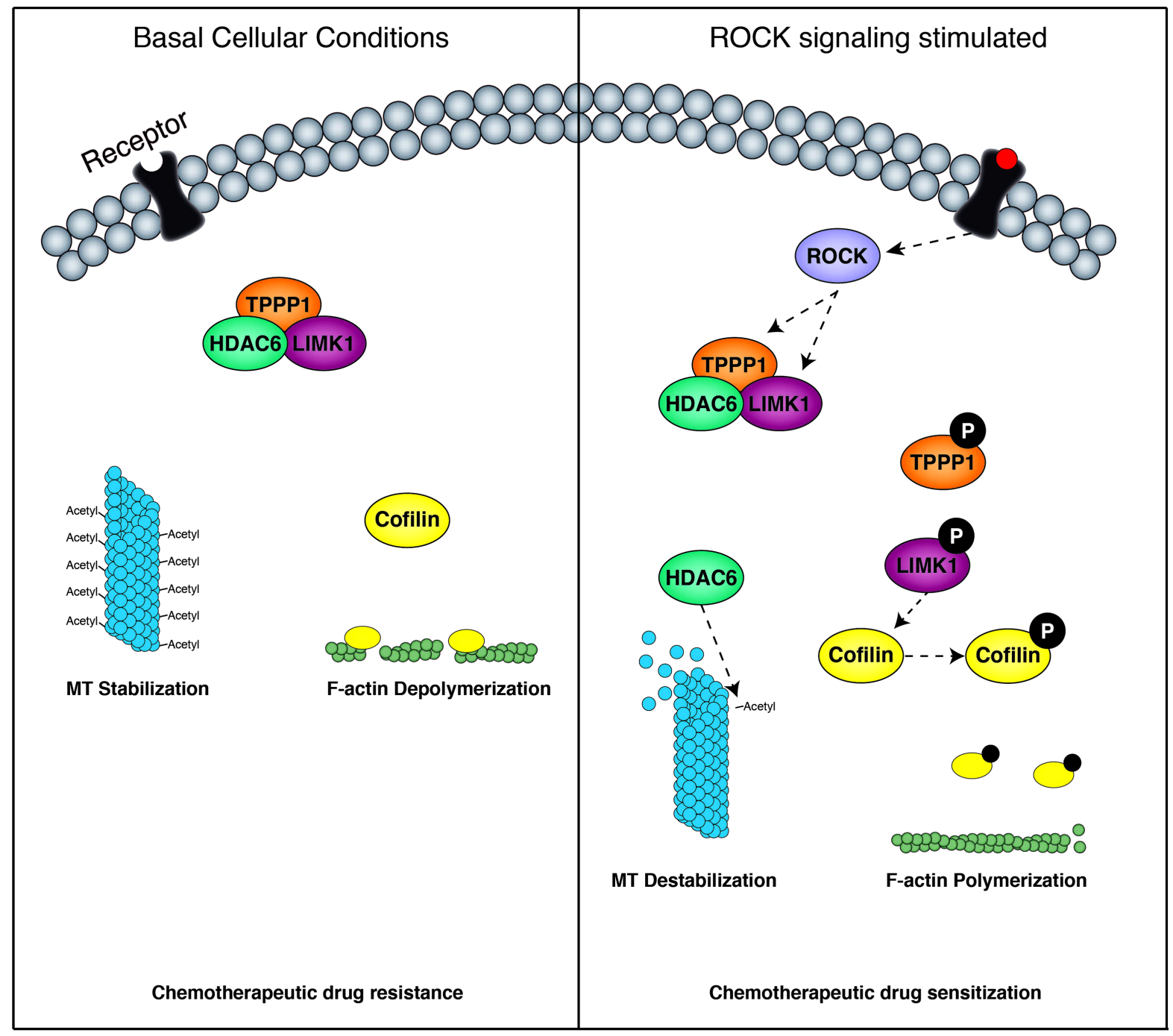

(a)

(b)

Figure 5. The LIMK1/TPPP1/HDAC6 complex regulates tubulin and actin stability in cells. (a) Under basal, unstimulated conditions, LIMK1, TPPP1 and HDAC6 form a trimeric complex. The complex formation results in inhibition of HDAC6 activity, resulting in increased tubulin acetylation and a consequential increase in tubulin rigidity. The complex reduces LIMK1-mediated phosphorylation of cofilin, leading to a reduction in F-actin in cells. (b) Upon ROCK activation, LIMK1 and TPPP1 are phosphorylated and the trimeric complex is disassembled. This drives an increase in HDAC6 activity and a subsequent reduction in tubulin acetylation and stability. Additionally, LIMK1 activity is increased resulting in increases in cofilin phosphorylation and F-actin levels in cells. 


\section{Acknowledgements}

We thank Drs Jian Li (MIPS, Monash University) and Kelly Rogers (Dynamic Imaging Facility, Walter and Eliza Hall Institute) for providing reagents or technical assistance and Dr Boris Sarcevic for critical reading of the manuscript.

\section{Author Contributions}

A.V.S and O.B conceived and designed the experiments. A.V.S and C.G performed the experiments. A.V.S, C.G and O.B analyzed the data. A.V.S and O.B contributed reagents, materials and analysis tools and wrote the manuscript.

\section{Funding}

This research was supported by grants and a fellowship to O.B from the National Health and Medical Research Council (NHMRC) and in part by the Victorian Government's Operational Infrastructure Support Program. A.V.S was the recipient of an Australian Postgraduate Award and a St Vincent's Institute Foundation Top-up Scholarship.

\section{References}

[1] Moriyama, K., Iida, K. and Yahara, I. (1996) Phosphorylation of Ser-3 of Cofilin Regulates Its Essential Function on Actin. Genes Cells, 1, 73-86. http://dx.doi.org/10.1046/j.1365-2443.1996.05005.X

[2] Nebl, G., Meuer, S.C. and Samstag, Y. (1996) Dephosphorylation of Serine 3 Regulates Nuclear Translocation of Cofilin. Journal of Biological Chemistry, 271, 26276-26280. http://dx.doi.org/10.1074/jbc.271.42.26276

[3] Ohashi, K., Nagata, K., Maekawa, M., Ishizaki, T., Narumiya, S. and Mizuno, K. (2000) Rho-Associated Kinase ROCK Activates LIM-Kinase 1 by Phosphorylation at Threonine 508 within the Activation Loop. The Journal of Biological Chemistry, 275, 3577-3582. http://dx.doi.org/10.1074/jbc.275.5.3577

[4] Maekawa, M., Ishizaki, T., Boku, S., Watanabe, N., Fujita, A., Iwamatsu, A., et al. (1999) Signaling from Rho to the Actin Cytoskeleton through Protein Kinases ROCK and LIM-Kinase. Science, 285, 895-898. http://dx.doi.org/10.1126/science.285.5429.895

[5] Sumi, T., Matsumoto, K. and Nakamura, T. (2001) Specific Activation of LIM Kinase 2 via Phosphorylation of Threonine 505 by ROCK, a Rho-Dependent Protein Kinase. The Journal of Biological Chemistry, 276, 670-676. http://dx.doi.org/10.1074/jbc.M007074200

[6] Acevedo, K., Li, R., Soo, P., Suryadinata, R., Sarcevic, B., Valova, V.A., et al. (2007) The Phosphorylation of p25/ TPPP by LIM Kinase 1 Inhibits Its Ability to Assemble Microtubules. Experimental Cell Research, 313, 4091-4106. http://dx.doi.org/10.1016/j.yexcr.2007.08.012

[7] Heng, Y.W., Lim, H.H., Mina, T., Utomo, P., Zhong, S., Lim, C.T., et al. (2012) TPPP Acts Downstream of RhoAROCK-LIMK2 to Regulate Astral Microtubule Organization and Spindle Orientation. Journal of Cell Science, 125, 1579-1590. http://dx.doi.org/10.1242/jcs.096818

[8] Gamell, C., Schofield, A.V., Suryadinata, R., Sarcevic, B. and Bernard, O. (2013) LIMK2 Mediates Resistance to Chemotherapeutic Drugs in Neuroblastoma Cells through Regulation of Drug-Induced Cell Cycle Arrest. PLoS One, 8, e72850. http://dx.doi.org/10.1371/journal.pone.0072850

[9] Schofield, A.V., Steel, R. and Bernard, O. (2012) ROCK Controls Microtubule Dynamics in a Novel Signaling Pathway that Regulates Cell Migration. The Journal of Biological Chemistry, 287, 43620-43629. http://dx.doi.org/10.1074/jbc.M112.394965

[10] Lehotzky, A., Tirian, L., Tokesi, N., Lenart, P., Szabo, B., Kovacs, J., et al. (2004) Dynamic Targeting of Microtubules by TPPP/p25 Affects Cell Survival. Journal of Cell Science, 117, 6249-6259. http://dx.doi.org/10.1242/jcs.01550

[11] Tokesi, N., Lehotzky, A., Horvath, I., Szabo, B., Olah, J., Lau, P., et al. (2010) TPPP/p25 Promotes Tubulin Acetylation by Inhibiting Histone Deacetylase 6. The Journal of Biological Chemistry, 285, 17896-17906. http://dx.doi.org/10.1074/jbc.M109.096578

[12] Foletta, V.C., Moussi, N., Sarmiere, P.D., Bamburg, J.R. and Bernard, O. (2004) LIM Kinase 1, a Key Regulator of Actin Dynamics, Is Widely Expressed in Embryonic and Adult Tissues. Experimental Cell Research, 294, 392-405. http://dx.doi.org/10.1016/j.yexcr.2003.11.024

[13] Arber, S., Barbayannis, F.A., Hanser, H., Schneider, C., Stanyon, C.A., Bernard, O., et al. (1998) Regulation of Actin Dynamics through Phosphorylation of Cofilin by LIM-Kinase. Nature, 393, 805-809. http://dx.doi.org/10.1038/31729 
[14] Fischle, W., Emiliani, S., Hendzel, M.J., Nagase, T., Nomura, N., Voelter, W., et al. (1999) A New Family of Human Histone Deacetylases Related to Saccharomyces cerevisiae HDA1p. The Journal of Biological Chemistry, 274, 11713-11720. http://dx.doi.org/10.1074/jbc.274.17.11713

[15] Acevedo, K., Moussi, N., Li, R., Soo, P. and Bernard, O. (2006) LIM Kinase 2 Is Widely Expressed in All Tissues. Journal of Histochemistry \& Cytochemistry, 54, 487-501. http://dx.doi.org/10.1369/jhc.5C6813.2006

[16] O’Reilly, L.A., Print, C., Hausmann, G., Moriishi, K., Cory, S., Huang, D.C., et al. (2001) Tissue Expression and Subcellular Localization of the Pro-Survival Molecule Bcl-w. Cell Death \& Differentiation, 8, 486-494. http://dx.doi.org/10.1038/sj.cdd.4400835

[17] Heng, Y.W., Lim, H.H., Mina, T., Utomo, P., Zhong, S., Lim, C.T., et al. (2012) TPPP Acts Downstream of RhoAROCK-LIMK2 to Regulate Astral Microtubule Organization and Spindle Orientation. Journal of Cell Science, 125, 1579-1590. http://dx.doi.org/10.1242/jcs.096818 